\title{
La Educación Social y la Educación Ambiental: dos Ámbitos Convergentes para la Acción Socioeducativa Ambiental
}

\author{
The Social Education and the Environmental Education: two Convergent Areas for the \\ Action Environmental Social Educational
}

\section{A Educação Social e a Educação Ambiental: dois Âmbitos Convergentes para a Ação Socioeducativa Ambiental}

\author{
Macarena Esteban Ibáñez ${ }^{1}$ \\ Daniel Musitu Ferrer ${ }^{2}$ \\ Luis Vicente Amador Muñoz ${ }^{3}$
}

\begin{abstract}
Resumen
Este artículo tiene como objetivo reflexionar sobre el papel que tiene la educación ambiental como un ámbito de desarrollo, aplicación y trabajo de la educación social desde un punto de vista teórico, a la vez que analiza aquellos ámbitos en las que pueden converger, en algunas ocasiones, y encontrarse alejadas, en otras. Ambos conceptos, se han venido estudiando como dos aspectos diferentes, con ciertos puntos en común, pero con metodologías y estrategias separadas e inconexas. Nuestro estudio considera que es necesario comenzar por construir un modelo, centrado en lo que hemos querido denominar intervención educativa socioambiental, que aúne ambas intervenciones. Desde esta perspectiva, toda intervención ambiental debe tener en cuenta los aspectos educativos y sociales porque las personas, los grupos y las comunidades son los afectados y beneficiarios de las transformaciones en el entorno físico, y toda intervención socioeducativa debe contemplar los aspectos ambientales implicados.
\end{abstract}

Palabras Clave: Educación Social. Educación Ambiental. Intervención Educativa Socioambiental.

\begin{abstract}
The goal of this article is reflect on the role of environmental education as a field of development, application and work of social education from a theoretical point of view, at the same time that it analyzes those areas in which they can converge in some occasions and to be distanced in others. Both concepts have been studied as two different aspects, with certain points in common, but with separate and disjointed methodologies and strategies. Our study considers that it is necessary to start by constructing a model, centered on what we have called socio-environmental educational intervention, which brings together both interventions. From this perspective, every environmental intervention must consider the educational and social aspects because individuals, groups and communities are affected and beneficiaries of the transformations in the physical environment and any socio-educational intervention must contemplate the environmental aspects involved.
\end{abstract}

Keywords: Social education. Environmental education. Socio-environmental educational intervention.

\section{Resumo}

Este artigo tem como objetivo refletir sobre o papel que a educação ambiental como uma área de desenvolvimento, implementação e trabalho de educação social, a partir de um ponto de vista teórico, ao analisar as áreas onde podem convergir, em algumas ocasiões, e se distanciar, em outras. Ambos os conceitos tem sido estudados como dois aspectos diferentes, com alguns pontos em comum, mas com metodologias e estratégias distintas e desconexas. Nosso estudo considera que é necessário começar a construir um modelo, focado no que denominamos intervenção educativa socioambiental, que combina ambas as intervenções. Nessa perspectiva, toda intervenção ambiental deve considerar

${ }^{1}$ Professora da Universidade Pablo de Olavide, Sevilha, Espanha, mestiba@upo.es

${ }^{2}$ Licenciado en Ciencias Biológicas pela Universidad de Valencia, Professor de Ciências no Colegio San Vicente de Paúl (Alcoy) e Mestrando em Educação Ambiental pela Universidade Pablo de Olavide. dani.musitu@colegiopaulalcoy.com

${ }^{3}$ Professor da Universidade Pablo de Olavide, Sevilha, Espanha, 1vamador@upo.es 
os aspectos educacionais e sociais, pois pessoas, grupos e comunidade são afetados e beneficiários das mudanças no ambiente físico, e toda intervenção socioeducativa deve contemplar os aspectos ambientais nela envolvidos.

Palavras-chave: Educação Social. Educação ambiental. Intervenção educativa socioambiental.

\section{Introducción}

La importancia de los elementos del ambiente, en la evolución y sostenimiento de la vida en el planeta, fue reconocida mucho tiempo antes de que se tomara plena conciencia de los posibles efectos negativos que su deterioro traería a la humanidad.

Desde mediados del siglo XX, el tema ambiental cobró dimensiones internacionales a partir del conocimiento y difusión de problemas asociados a la degradación del ambiente.

Existen muchos problemas ambientales que están enfermando a nuestro planeta. Estos, a su vez, guardan estrecha relación con otros problemas económicos y sociales.

Los problemas, desde una perspectiva global, como expone Cuello-Gijón (2003), se caracterizan por:

- Ser persistentes.

- Estar en continuo aumento.

- Ser, en la mayoría de los casos, de difícil reversibilidad.

- Responder a múltiples factores, y, en ellos, se entrelazan aspectos de diversa naturaleza: ecológicos, económicos, sociales, culturales, éticos etc.

- Tener consecuencias más allá del tiempo y el espacio donde se generan.

- Ser parte de otro problema más complejo y, a la vez, suma de numerosos y pequeños problemas.

- Tener soluciones complejas y múltiples que, a veces, dependen de muchas pequeñas soluciones.

- Las soluciones de tipo legal, correctivo, coercitivo, disuasorio etc., no son eficaces por sí mismas.

- Las soluciones de tipo tecnológico, en el mejor de los casos, sólo palian los efectos, es decir tratan el proceso en sus fases finales (medidas compensatorias, correctivas, pocas veces anticipatorias o preventivas).

Los impactos ambientales y sociales que dan origen a los problemas surgen de muchos factores, como: niveles insostenibles de consumo de recursos, tecnologías insuficientes, prácticas administrativas inapropiadas, políticas asimétricas de desarrollo económico, leyes y regulaciones que ignoran la verdadera exposición de los seres humanos e innumerables procesos políticos no participativos. Los patrones de consumo de todas las comunidades, tanto ricas como pobres, se asocian en conjunto con amenazas críticas al ambiente como son el deterioro de la salud humana (MUÑOZ; IBAÑEZ 2011).

La mayoría de los países padecen de una serie de problemas ambientales, de variada naturaleza, asociados a diversas actividades humanas, que ponen en peligro el desarrollo sustentable del país. Entre ellos, destacamos algunos ejemplos: el nivel de contaminación atmosférica, el volumen de residuos urbanos e industriales y la congestión vehicular, entre otros. Se debe tener en cuenta la opinión de María Neira, Directora de Salud Pública y Medio Ambiente de la Organización Mundial de la Salud (OMS, 2013, p.2), quién expreso:

En cuanto a factores ambientales, buenas inversiones en los mismos, hacer accesibles a toda la población el agua, el saneamiento y la higiene, podría reducir un 25 por ciento la morbimortalidad en el mundo y disminuir las enfermedades más prevalentes, ya que las diarreicas siguen matando a una gran cantidad de niños. Parece absurdo que en el siglo XXI sigamos hablando de que un determinante fundamental de la salud es tener acceso al 
agua y al saneamiento cuando el gran cambio en la situación epidemiológica del mundo occidental se produjo precisamente cuando se entendió que había que invertir en esto. Respecto a los países desarrollados, uno de los factores ambientales que podrían tener un gran impacto en la prevención de la enfermedad es la contaminación ambiental, responsable de un número elevado de muertes cada año y, sobre todo, de patologías crónicas y agudas, especialmente respiratorias y cardiovasculares.

Con la acumulación de múltiples fracasos en la solución de los problemas ambientales ha quedado claro que su enorme complejidad obliga a los diversos sectores involucrados a colaborar muy estrechamente. Los grupos gubernamentales y académicos están en un sitio de particular responsabilidad en este sentido. Consideramos que la principal solución a los problemas ambientales es la educación ambiental en todos los niveles y sectores de la sociedad, productivos o no. Esta es una herramienta fundamental para lograr un cambio de actitud y de comportamiento en la sociedad, no sólo en los productores sino también en los consumidores de todo el mundo.

Los cambios de actitud solamente se producen cuando existe información suficiente y cuando se conoce el verdadero alcance del deterioro ambiental. Como expone Aramburu (2001), la gran mayoría de la población desconoce o no considera preocupantes las denuncias de los ecologistas; no se sienten responsable de ellos y no saben cómo actuar, si deciden adoptar actitudes positivas ante la naturaleza.

Por ello es necesario un mayor esfuerzo de la educación como ya se auguró en postulados de la Declaración sobre el Medio Humano en la Conferencia de Estocolmo del año 1972, según explica Gutiérrez-Pérez (1995, p. 87):

Es indispensable una labor de educación en cuestiones ambientales, dirigida tanto a las generaciones jóvenes como a los adultos, y que preste la debida atención al sector de la población menos privilegiado, para ensanchar las bases de una opinión pública bien informada y de una conducta de los individuos, de las empresas y de las colectividades inspirada en el sentido de responsabilidad en cuanto a la protección y mejoramiento del medio en toda su dimensión humana.

La educación es una responsabilidad social, pero se relega, generalmente, al ámbito familiar y al ámbito formal, reglado. La sociedad acepta que la educación formal siga teniendo un papel preponderante, y a la escuela se ha delegado la misión de formar unas generaciones comprometidas con nuevos sistemas de valores, con unos conocimientos sólidos sobre el medio, con el que deben establecer continua comunicación. El conjunto de intenciones y estrategias que desde años se han orientado en este sentido, han contribuido a designar el término Educación Ambiental, que España está asumiendo como acción prioritaria en el sistema educativo.

Según expresa Pastor (2000, p. 36), se trata de

Una Educación Ambiental que se constituye en un proceso que reconoce valores y aclara conceptos centrados en fomentar las actitudes, destrezas, habilidades y aptitudes necesarias para comprender y apreciar las interrelaciones entre el ser humano, su cultura y la interrelación con la naturaleza.

Además, reconocemos que la educación "es fundamental para adquirir conciencia, valores, técnicas y comportamientos ecológicos y éticos en consonancia con el desarrollo sostenible y que favorezcan la participación comunitaria efectiva en decisiones. Así lo afirma la conferencia de toma de decisiones (ONU, 1992, s/p). 


\section{La Educación Ambiental como herramienta social}

A tenor de lo expuesto en el apartado anterior, podemos plantear que la disyuntiva de explotar los recursos naturales o conservarlos intocables ha pasado, desde hace más de medio siglo a la fecha, de una discusión casi teórica hasta un serio enfrentamiento de puntos de vista sobre el estilo de vida de las actuales y futuras generaciones. La progresiva urbanización provocada por la concentración acelerada de la población y las modificaciones en las políticas relativas al campo se han traducido en cambios drásticos en el ambiente.

A causa de ello, podemos observar que ante la demanda creciente de productos agrícolas, pecuarios, forestales, mineros y energéticos, para la satisfacción de las necesidades urbanoindustriales, se alteran irreversiblemente los ecosistemas naturales. Aunque paradójico, la causa de la problemática señalada es la búsqueda del desarrollo, entendido, éste, en términos del bienestar de los individuos, como producto creciente de la posibilidad de acceder a las oportunidades para la satisfacción de sus necesidades, tanto en los aspectos económicos como políticos y sociales.

Es innegable que el origen principal de la problemática ambiental lo encontramos en la conducta humana. La falta de información, conciencia y capacidad se constituyen en las principales causales de la inadecuada respuesta social. Para dimensionar adecuadamente lo expuesto, justo es señalar que la crisis del medio ambiente no es un problema moderno, lo nuevo son sus dimensiones. A esto cabe acotar que la tecnología propicia la degradación acelerada del medio ambiente y los recursos naturales, cuando no es usada racionalmente, y que la capacidad de respuesta orientada a su restauración se sitúa en el largo plazo.

Hablar de sustentabilidad en el desarrollo implica cuestiones muy amplias, obligándonos a reflejar sobre cómo ocurriría una relación armónica que contemplara las necesidades de supervivencia humana sin despreciar la naturaleza. Todavía, ¿cuál sería este nivel de vida humano adecuado?, ¿cómo medir lo que es o no apropiado en una sociedad de consumo? Tales cuestiones pueden asumir facetas muy subjetivas, por lo que no hay respuestas simples.

Esas discusiones surgen en el ámbito de lo planteado en el documento de la Constitución Española, que expone que todo individuo tiene derecho a disfrutar de un medio ambiente adecuado y el deber de conservarlo. Así mismo, los poderes públicos velarán por la utilización racional de todos los recursos naturales, con el fin de proteger y mejorar la calidad de vida y defender y restaurar el medio ambiente, apoyándose en la colectividad (ESPAÑA, 1978).

Cotidianamente, se señala que la conservación y el desarrollo raramente han sido combinados y, casi siempre, parecen incompatibles, y lo serán en la medida en que no se haya previsto un desarrollo sostenido. Bajo esa perspectiva hay que considerar que el bienestar social se sustenta en el aprovechamiento de los recursos naturales y en la modificación de la biosfera, acordes al uso los recursos humanos y financieros, de tal forma que la satisfacción de las necesidades y el nivel de vida dependen, directamente, del modelo de desarrollo que se adopte.

Queda claro, entonces, que el modelo de desarrollo que se ha venido aplicando resulta atentatorio para los recursos naturales, los que junto al medio ambiente se han sometido a una presión y ritmo de consumo superior a su capacidad de regeneración. Se hace necesario, entonces, partir de bases diferentes que contemplen como metas no sólo el bienestar de las actuales generaciones, sino que prevea la sana subsistencia de las futuras. Por lo tanto, el modelo de desarrollo socioeconómico que se requiere es un modelo de desarrollo sustentable, en el cual es fundamental que el aprovechamiento y uso de los recursos naturales sea racional y potencialmente sostenible.

En este sentido es que se manifiesta una nueva cultura ecológica que se hace patente en todos los niveles y sectores. Mientras que en el gubernamental se percibe un creciente proceso de 
organización, en la sociedad civil se incrementa el reclamo, la denuncia y la gestión como expresiones participativas. Lo mismo observamos la creación de dependencias oficiales, como la manifestación constante de organizaciones no gubernamentales, eventos políticos, científicos etc.

La relevancia de la Educación Ambiental como campo de conocimiento teórico y práctico es claramente reconocida, en la actualidad, habida cuenta las variables económicas, políticas, sociales y culturales que permiten explicar las diversas situaciones que se manifiestan en el medio, que permiten hablar de problemática ambiental, así como de la necesidad de concertar alguna medida para afrontarla. En realidad, se espera que la Educación Ambiental sea capaz de suscitar cambios en las mentalidades, actitudes, saberes, comportamientos etc., que han de tener las personas y sociedades, cada vez más inquietas por la exigencia de conjugar la vida diaria con los gigantes cambios tecnológicos, económicos, culturales, ambientales en los que se expresa la modernidad: con los efectos y consecuencias que están produciendo en las sociedades actuales, desarrolladas y no desarrolladas, y en su ciudadanía.

En este sentido, preguntamos sobre el papel que juega la Pedagogía Social en la comprensión del medio: ¿qué puede hacer la Educación Social como práctica y como profesión a favor del medio ambiente?

Desde finales del siglo XX, en torno a la Educación Ambiental, se sucedieron conferencias (Estocolmo, 1972; Belgrado, 1975; Tblisi, 1977 etc.); además de informes (Meadows, 1972; Brundtlad, 1987) y la aparición de las grandes organizaciones ecologistas como Greenpeace y World Wide Fund for Nature - WWF, mientras en España se desarrollan las Jornadas de Educación Ambiental (Sitges, 1983) y avanzan las Cumbres de la Tierra (Río de Janeiro, 1992). Todo ello va provocando que la ciudadanía y la clase política fueran tomando conciencia de los límites del planeta (RODRIGO-CAÑO, 2019).

En España, la Educación Ambiental, tras el apogeo de los últimos años del siglo XX, culminaba con la publicación del Libro Blanco de la Educación Ambiental. Libro, este, que recoge los principios que deben guiar al desarrollo sostenible: la necesidad de conservar los recursos naturales. La existencia de límites físicos que hacen imposible el crecimiento sin fin. La necesidad de alcanzar objetivos sociales. La solidaridad intra e intergeneracional (MMA, 1999).

Dicho Libro Blanco, además de traer los principios básicos de la educación ambiental, hace una discusión de los instrumentos para implantarse programas de acción, propuestas de actuación en las comunidades, el papel que juega la educación ambiental en el sistema educativo, medios, en las empresas, sindicatos, organizaciones de la sociedad civil, en las varias esferas de los gobiernos etc (MMA, 1999).

El mismo Libro Blanco propone la educación ambiental como "estrategia imprescindible de acción para ofrecer resoluciones a la problemática de la degradación medioambiental" (MMA, 1999, p.8). La Educación se hace eco de estos cambios y viene a exponerlos y, entre ellos, aparece el crecimiento y desarrollo sostenible y protección del medio ambiente, sin olvidarnos de todos los que recoge, entre otros: atención a la diversidad y exclusión; internalización, globalización económica y socio-económica y la necesidad de respuesta local y atención a los servicios a la ciudadanía que ello demanda; el nuevo marco internacional que conlleva el contacto y el intercambio entre colectivos, ciudadanos con identidades diversas. Demanda de una nueva cultura de la interculturalidad, la mediación y la convivencia.

Todo ello se traduce en un nuevo tipo de sociedad, la sociedad del conocimiento. En ella aparecen dos perfiles educativos claves: el aprendizaje dinámico y el de aprendizaje para toda la vida, vinculados, de manera transversal, a la incorporación y uso de las nuevas tecnologías de la información y la comunicación y a la participación crítica y responsable para reaccionar ante las transformaciones y los desajustes que la nueva situación produce. 
Y, a partir de aquí, expone acciones, planes, programas y proyectos que desde una visión educativa estarían relacionados y unidos a acciones continuas, en donde la implicación del profesional de la educación y de los participantes es absolutamente fundamental (MUÑOZ; IBAÑEZ, 2011):

- Programas, acciones, proyectos e iniciativas vinculadas a la vida diaria.

- Programas, acciones, proyectos e iniciativas vinculadas a la mejora de la calidad de vida

- Programas, acciones, proyectos e iniciativas culturales y de ocio.

- Programas, acciones, proyectos e iniciativas vinculadas al medio ambiente.

Por esto, el principal reto de la EA en España sigue siendo, hoy, traspasar los límites de la educación reglada y alcanzar, de modo efectivo, al resto de colectivos sociales. Ardua tarea la que tienen todos los agentes implicados, entre ellos, los educadores/as sociales para poder analizar qué podemos hacer para solucionar esta necesidad. Ya que no puede delegarse únicamente en los más jóvenes la responsabilidad de afrontar cuanto antes un progreso equilibrado en el ámbito social, económico y medioambiental. Nos parece una realidad actual contemplar que aún queda mucho por hacer en el desarrollo de la EA y que, además, debe de seguir aspirando a llegar a todas las edades. Se trata de una educación permanente, que se desarrolle a lo largo de la vida, sin discriminar ninguna etapa evolutiva, ya que en cada una de ellas podemos trabajar en una dirección u otra.

\section{Educación Social versus Educación Ambiental}

Es en este campo en donde destaca la importancia del papel de la educación que debe traspasar los ámbitos de la educación reglada, formal, escolar, llegando al marco del desarrollo socioeducativo de personas y colectivos en el contexto de la educación no reglada, no formal. Es aquí en donde aparece la Educación Social, desde la vertiente académica y profesional dando respuesta a las demandas sociales y de ciudadanía y, por supuesto, desajustes que vienen a provocar esas transformaciones.

Según explanan Viana-Orta, Francés y Zayas (2018), la educación social, en España, queda aglutinada a disciplina trabajo social. Es facto que, en Europa, las fronteras entre Pedagogía Social, Trabajo Social y Educación Social no son nítidamente delimitadas, tampoco los perfiles profesionales vinculados a ellos. Así:

En el marco europeo existe una gran diversidad que dificulta tener conceptos, formaciones y perfiles profesionales compartidos en el campo de la intervención social. Así, existe una enorme diversidad terminológica e idiomática; diversidad de ámbitos de trabajo; diversidad de usuarios; diferentes miradas y fundamentos teóricos; diversidad de respuestas dadas a las cuestiones sociales en cada lugar (VIANA-ORTA; FRANCÉS; ZAYAS, 2018, p. 2).

En España, el Real Decreto 1420, de 30 de agosto de 1991, estableció el título universitario oficial del Diplomado en Educación Social, bien como las directrices propias de los planes de estudio conducentes a la obtención del mismo. Además, en sus términos, el Real Decreto 1420 explana que "las enseñanzas deben orientar-se a la formación de un educador en los campos de la educación no formal, educación de adultos (incluidos los de la tercera edad), inserción social de personas desadaptadas o minusválidas, así como en la acción socioeducativa" (ESPAÑA, 1991, p. 24669, grifos nuestros).

Está claro que la educación ambiental puede relacionarse con la educación social en el aspecto que implica las llamadas acciones socioeducativas. 
La figura y profesión de Educador/a Social son conceptos bastante complejos y en algunos casos muy desconocidos. Es una profesión que se ha ido concretando en el tiempo, antes mismo de la creación del grado universitario por el Decreto Real 1420/1991, con el fin de dar respuesta a las necesidades socioeducativas de los individuos y grupos sociales. En este proceso, se hizo evidente la importancia de su actividad profesional, que traspasa e incluso complementa los ámbitos de la educación reglada o escolar. Al mismo tiempo, acerca el desarrollo e intervención socioeducativa a personas y colectivos con la finalidad de promover el cambio social desde lo educativo.

Por ello, podemos decir que es un campo en permanente construcción dada la necesidad que tiene de responder a los cambios de la propia sociedad, a los contextos, tanto colectivos como personales, imbricándose con diversas otras áreas del conocimiento humano, incluso la educación ambiental.

Con la Ley Orgánica 11/1983, de 25 de agosto, y el Real Decreto 1497/1987, de 27 de noviembre se establecen las directrices generales que permitieron la elaboración de los planes de estudio de la diplomatura de Educación Social. Ello conllevó la consolidación inicial de esta titulación a nivel académico, social y profesional (ESPAÑA, 1983, 1987, 1991).

Con ello, se pretende propiciar la consecución por los estudiantes de una formación universitaria que, además de propiciar los conocimientos que le son imprescindibles y necesarios para dar respuesta a su formación integral, incluyendo las capacidades, competencias imprescindibles para el desarrollo profesional, para su incorporación al ámbito laboral (VIANAORTO; FRANCÉS; ZAYAS, 2018).

En definitiva, podríamos considerar al educador/a social como la persona que, después de una formación específica, favorece, mediante métodos y técnicas pedagógicas, psicológicas y sociales, el desarrollo personal, la maduración social y la autonomía de las personas, jóvenes o adultos incapacitados o desadaptados o en peligro de serlo, segundo disponen las asignaturas del grado académico (VIANA-ORTO; FRANCÉS; ZAYAS, 2018).

Es un profesional especializado que interviene en procesos de acción socioeducativa para modificar situaciones de personas, grupos o comunidades, a través de estrategias y recursos adecuados. Que vienen a llevar a cabo funciones propias del proceso de atención global (evaluación, intervención, inserción) en todos los contextos (individual, familiar, social e institucional) con el soporte de programas individualizados. En este campo, y bajo estos criterios, es en donde ponemos en valor la relación, la vinculación entre dos realidades que transcurren, en ocasiones paralelas a lo largo del tiempo y que terminan compartiendo criterios, finalidades, compromisos etc. (MUÑOZ et al., 2014).

Tal y como se hacen eco Caride y Meira y (2001), ambas se desarrollan de forma definitiva en el periodo del estado de bienestar, con el fin de dar respuesta a los compromisos que plantean los nuevos desajustes sociales propios de las transformaciones del momento. En un caso, como consecuencia de los desequilibrios emanados, como consecuencia de los excesos industriales y los problemas que plantea el modelo socioeconómico. En el otro, alarmada por las disfunciones sociales que provoca; siendo aquí, como hemos visto anteriormente, en donde la Educación Social termina haciéndose eco y tratando de dar respuesta. Compartiendo ambas la crisis global y la necesaria seguridad ante la calidad de vida y la incertidumbre que, en cualquier caso, la sociedad actual conlleva.

Los iniciales planteamientos educativos surgen en el contexto de formar en valores que faciliten el respeto a la naturaleza, en donde las personas realizamos nuestras actuaciones. A este planteamiento en un principio reduccionista (didáctica de la ecología), sucede otro más inter o multidisciplinar, que da más importancia a la naturaleza social y cultural del medio ambiente. Esta perspectiva queda de manifiesto en la Figura 1. 


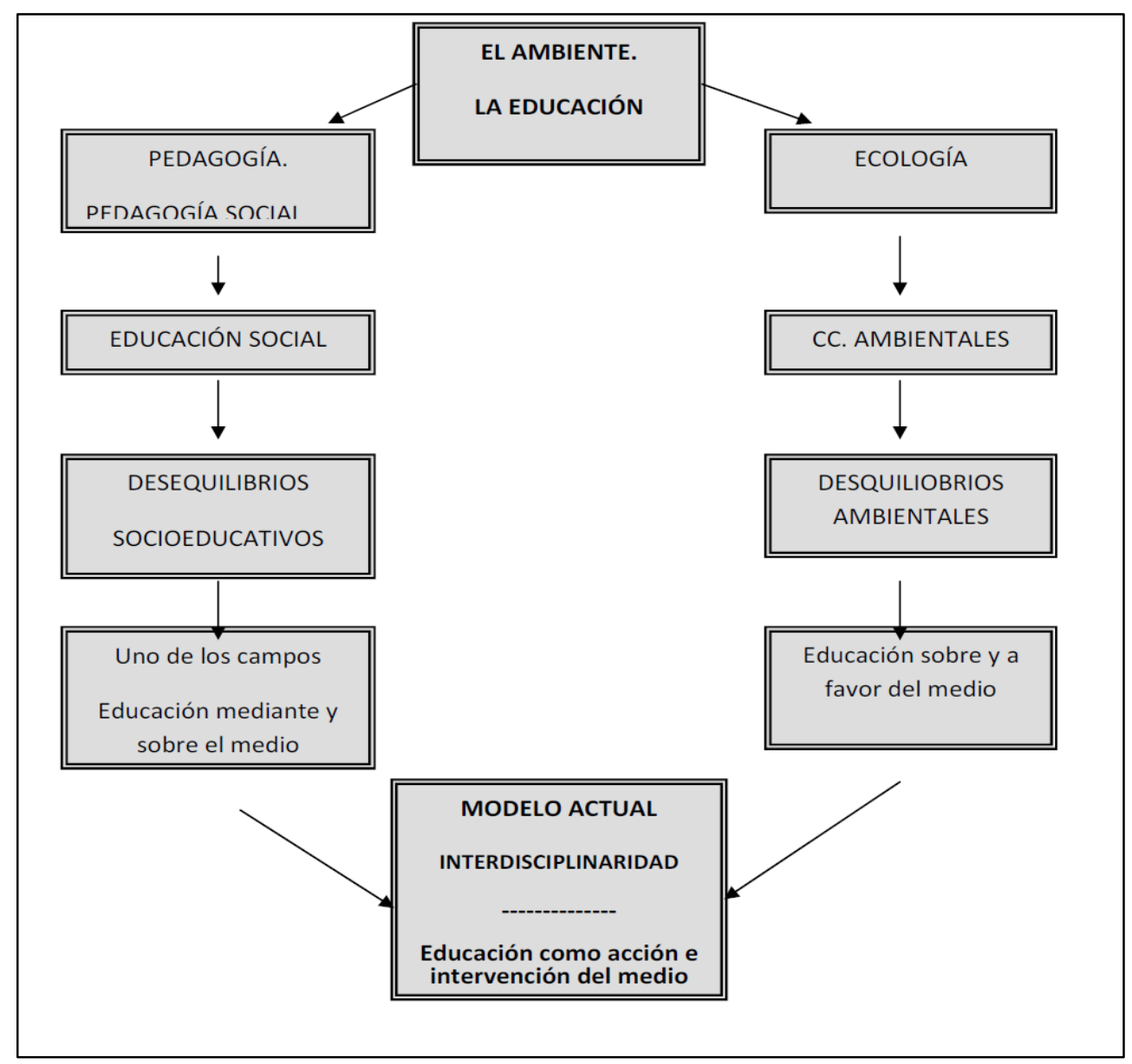

Figura 1 - Relaciones entre Medio Ambiente y Educación Fuente: elaboración propia a partir de Rodríguez (2005)

Vemos como con el devenir, con el proceso ambiental y educativo, en donde se aprecia que la Ecología y la Educación Social, como praxis de la Pedagogía Social, da lugar a una nueva visión a la que denominamos Educación Ambiental. Dado que nace como un elemento integrador, autoras como Rodríguez (2005) viene a denominarla Educación Socioambiental.

Bien entendido que la Educación Social, además de configurarse como una disciplina científica, es un hecho, un fenómeno, una acción y por ello puede ser objeto de estudio y análisis, pero no la ciencia misma. Así, la formación en educación social debe abordarse de manera interdisciplinar y desde diferentes ámbitos de las ciencias socioeducativas ya existentes.

Hay que entender el medio ambiente como un conjunto de representaciones, dimensiones interrelacionadas y complementarias, que educativamente debe concebirse como un proyecto social y comunitario. En dicho ambiente, como se expone en la Figura 2, se desarrollarán las actividades educativas; al tiempo que, con el compromiso que debe ser el eje que guie las mismas, se debe fomentar la participación, el desarrollo comunitario etc. (SAUVÉ, 2006). Véase la Figura 2: 


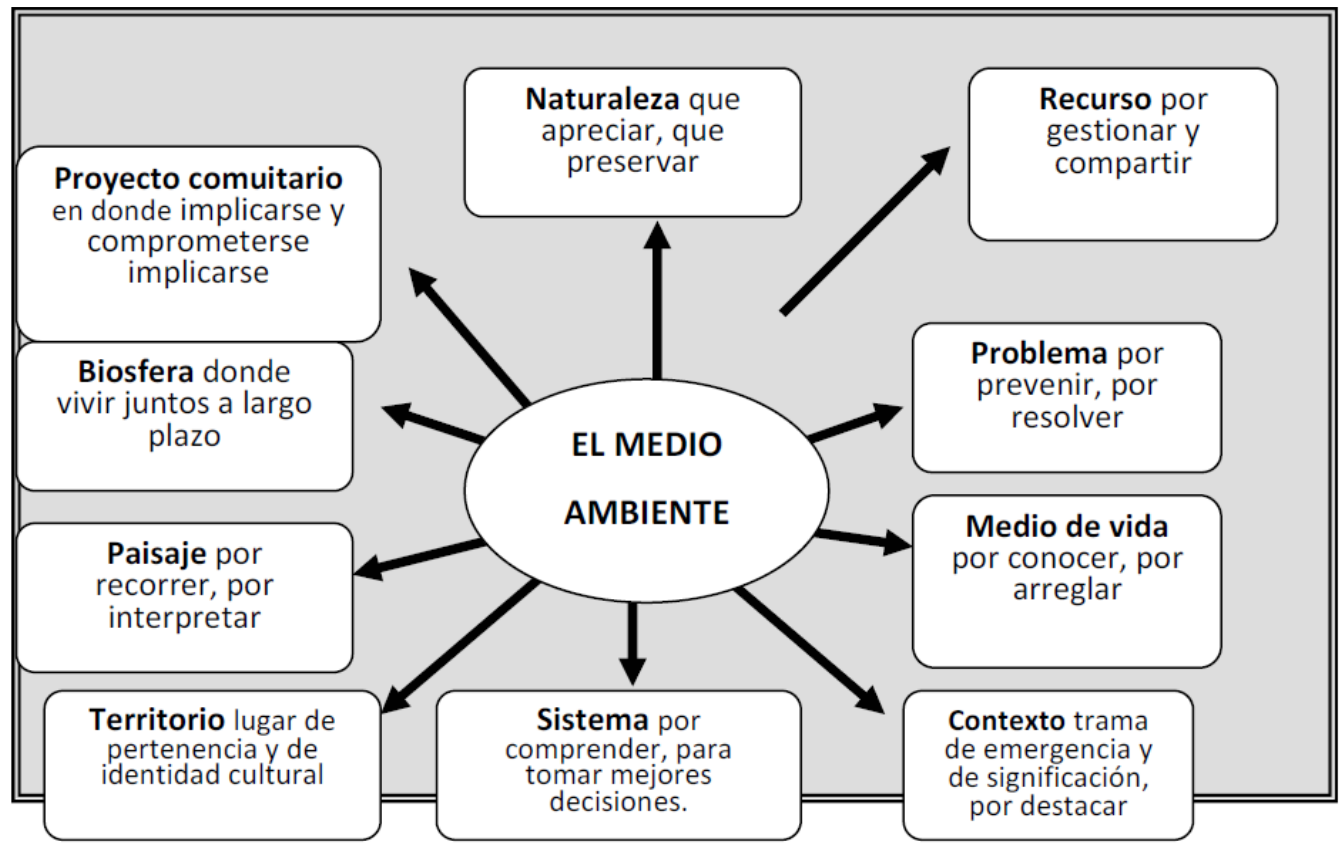

Figura 2 - Representaciones del Medio Ambiente

Fuente: los autores

Con ello se busca asegurar los medios que permitan llevar una vida comunitaria correcta y adecuada, en donde la educación, incluida la ambiental, no debe ser entendida como resolución de problemas o modificación de conducta cívicas.

La Educación Social y la Educación Ambiental aparecen como intervención socioeducativa y ambiental, como praxis, con finalidades idénticas a niveles teóricos, objetivos, metodología y, en muchas ocasiones, contenidos compartidos. Lejos de mantener líneas separadoras, aparecen interacciones e interdisciplinaridad que va a enriquecer y favorecer la comprensión de la crisis ambiental, de carácter social, que es el origen de los desequilibrios ambientales que faciliten la toma de decisiones superadoras de los mismos (CARIDE; MEIRA, 2001).

Esta forma de entender la Educación Ambiental, dentro del contexto de la Educación Social, tiene la ventaja de entenderse, al tiempo que facilita respuestas a las necesidades de sostenibilidad. Todo ello, dentro del bienestar general en un medio ambiente equilibrado que viene a facilitar el desarrollo y el progreso de la vida (RODRÍGUEZ, 2005).

Analizar las distintas perspectivas de la Educación Ambiental y de la Educación Social consiste en reflexionar sobre cómo el entorno influye en los procesos de acceso individual a la cultura y, también, de cómo los individuos, dentro de una sociedad, influyen en el Medio en que se desenvuelven al mismo tiempo que están influidos por éste. Es decir, supone tener en cuenta las complejas interacciones Individuo-Medio.

En sociedades como la nuestra, el Medio Ambiente no es sólo lo que de natural hay en el entorno de los individuos, sino que también es el resultado de las acciones de estos individuos en dicho medio natural, y esto es algo a tener muy en cuenta a la hora de elaborar el marco conceptual de la Educación Ambiental. Por ello, no podemos olvidar que cuando se habla de EA no estamos abordando sólo un aspecto del entorno (medio natural) en el que los individuos se educan. Este planteamiento, demasiado frecuente, empobrece el análisis y restringe en demasía la visión completa de la realidad socioeducativa. 
A tenor de lo expuesto, la educación social tiene que ver con la participación ciudadana en la realidad social, la cual envuelve las relaciones de los individuos con el medioambiente. Según el Libro Blanco de Educación Ambiental de España:

La participación ciudadana en los planes y actuaciones ambientales tiene, en sí misma, un fuerte componente educativo, de concienciación, de aprendizaje de destrezas y de compromiso. Pero requiere tiempo, predisposición, responsabilidad y actitud abierta. Exige, además, un aprendizaje no sólo de habilidades sociales sino también de métodos (MMA, 1999, p. 33).

Donde se percibe que hay una interrelación entre las propuestas de la educación social y aquellas de la educación ambiental. Merece la pena citar esas recomendaciones en su totalidad, porque recogen, con exhaustividad, ese giro tan necesario para renovar una acción educativa, en general poco rigurosa, que se ha recluido en la actividad extraescolar de la enseñanza reglada y en la animación sociocultural de la infancia (CALVO; CORRALIZA, 1994), o se ha diluido en el mercado del ocio y en la oferta sensibilizadora de los medios de comunicación; y tan necesario, al mismo tiempo, para reconvertirle en un destino propicio para la intervención socioeducativa en espacios comunitarios, en directa conexión con la cuestión de desarrollo. Estas recomendaciones sobre la nueva Educación Ambiental son entre otras, en la visión de Faraco (2001):

- Mayor atención a los programas educativos y a los factores sociales.

- Incidencia en el cambio de valores y de comportamientos.

- Progresiva extensión de la Educación Ambiental al conjunto de la comunidad.

- Integración de la Educación Ambiental en todos los niveles de la política ambiental y en los programas de desarrollo.

- Fomento de la participación ciudadana en las iniciativas ambientales.

\section{Intervención Ambiental y Social: hacia una Intervención Educativa Socioambiental}

A tenor de lo expuesto, nuestra idea es que podamos llegar a un modelo común que aúne ambas intervenciones en las personas, las comunidades y los entornos, constituyendo una unidad global inseparable, con estrechas interrelaciones entre las partes que pasan a definirse mutuamente para configurar una estructura integrada. Desde esta perspectiva, sugerimos que toda intervención ambiental tiene que tener en cuenta los aspectos educativos y sociales, puesto que, en último término, las personas, los grupos y las comunidades son los afectados y beneficiarios de las transformaciones en el entorno físico.

De igual forma, toda intervención socioeducativa tiene que contemplar los aspectos ambientales implicados, puesto que esta intervención siempre se desarrolla en un contexto sociofísico determinado. En este contexto, la educación social y la educación ambiental devienen elementos clave por armonizar la relación entre el desarrollo y la transformación del entorno y el desarrollo personal y social de las personas; en definitiva, lo que desde una concepción amplia podemos denominar desarrollo educativo/socio/ambiental sostenible.

Así:

La intervención social tiene como finalidad el involucramiento de la sociedad en la construcción, diseño e implantación de políticas públicas estatales o comunitarias, así como también en su implementación y evaluación. En esta línea, y teniendo en cuenta la transversalidad del componente ambiental, dicha intervención social se constituirá en un instrumento esencial para introducir la temática ambiental en la intervención social ya que ésta permite confrontar problemas y conflictos ambientales (MUÑOZ; IBAÑEZ, 2011, p. 156). 
Por lo tanto, nuestra idea es que cuando tengamos que llevar a cabo intervenciones socioeducativas en relación con la temática ambiental, deberíamos centrarnos, principalmente, como reconocen Alvino y Sessano (2008), en intervenciones orientadas a la resolución de problemas. En ellas es necesario involucrar al ciudadano, ya que es éste el que participa tanto en la identificación de los mismos como, en la investigación y puesta en marcha de las conclusiones las que serán analizadas, debatidas y evaluadas técnica y éticamente. Finalmente, se extraen las ideas centrales que permitan tomar decisiones para la acción, que sean compatible con la preservación del ambiente y los intereses de la comunidad local (ALVINO; SESSANO, 2008).

A raíz de lo expuesto, consideramos que se hace necesario, empezar a hablar del concepto de intervención educativa socioambiental, que incluya a la Educación Ambiental como campo de conocimiento teórico y práctico, claramente reconocida, en la actualidad. Ello es consecuencia, principalmente, de las variables económicas, políticas, sociales y culturales que permiten explicar las diversas situaciones que se manifiestan en el medio, que posibilitan hablar de problemática ambiental, así como de la necesidad de concertar alguna medida para afrontarla.

A la larga, la Educación Ambiental debe ser capaz de suscitar cambios en las mentalidades, actitudes, saberes, comportamientos etc.... que han de tener las personas y sociedades, cada vez más inquietas por la exigencia de conjugar la vida diaria con los enormes y rápidos cambios tecnológicos, económicos, culturales y ambientales en los que se manifiesta nuestra sociedad, con todos sus efectos y consecuencias (SÁEZ, 2007).

Es en este ámbito en el que podemos decir que la Educación Social será aquella acción sistemática y fundamentada, de soporte, mediación y transferencia que favorezca el desarrollo de la sociabilidad del sujeto a lo largo de toda la vida, circunstancias y contextos, promoviendo su autonomía, integración y participación crítica, constructiva y transformadora en el marco sociocultural que le envuelve, contando, en primer lugar, con los propios recursos personales, tanto del educador, como del sujeto, y, en segundo lugar, movilizando todos los recursos socioculturales necesarios del entorno o creando, al fin, nuevas alternativas.

Podemos destacar por lo manifestado y por las diferentes aproximaciones conceptuales que existen dos grandes líneas en las aportaciones y aproximaciones a la conceptualización de Educación Social que redundan en la Educación Ambiental: las que ponen el énfasis en los ámbitos de trabajo, o los que se centran en los fines de la actuación como criterio. Es por ello queremos hacernos eco de la definición que realiza la Asociación Estatal de Educación Social - Asedes (2007), que viene a reconocer la relación existente entre la Educación Social y su relación con la temática ambiental. Es un derecho de la ciudadanía que se concreta en el reconocimiento de una profesión de carácter pedagógico, generadora de contextos educativos y acciones mediadoras y formativas, que son ámbito de competencia profesional del educador social, posibilitando:

- La incorporación del sujeto de la educación a la diversidad de las redes sociales, entendida como el desarrollo de la sociabilidad y la circulación social.

- La promoción cultural y social, entendida como apertura a nuevas posibilidades de la adquisición de bienes culturales, que amplíen las perspectivas educativas, laborales, de ocio y participación social.

En esta línea, cabría preguntarse ¿cuáles son las perspectivas profesionales de los Educadores/as Sociales en relación con la Educación Ambiental? Para ello, trabajamos con varias dimensiones:

- Desde el ámbito social, en cuanto a nichos de empleo y la necesidad de dar respuesta a nuevas demandas sociales. En este terreno, debemos hacernos eco de lo manifestado por la propia Comisión Europea que viene a determinar cuatro bloques: vida diaria, mejora de este marco de vida, ocio y cultura y medio ambiente, es evidente que en este campo cabría, a su 
vez, el determinar alguna sugerencia más en el campo de temas emergentes. Todos ellos tienen que ver con las nuevas necesidades sociales, información, sociedad del conocimiento y TIC, situaciones derivados de la migración, sectores con necesidades específicas, atención a la diversidad etc.

- Desde el criterio del estado de bienestar. Es evidente que el educador/a social se convierte en una figura que debe intentar la búsqueda de la garantía social y, por tanto, la integración. También, parece evidente que debamos seguir reclamando que sea el sector público el que lidere estos servicios hacia la comunidad, para que sean realmente los sectores y grupos que más lo necesiten, aquellos que se vean favorecidos por su actuación.

- Desde el campo social y colectivo. Desde el ámbito de la Educación Social se debe favorecerse la participación de los individuos, tanto en los temas sociales y colectivos como en los particulares. Tiene que propiciar que sean los propios usuarios, la ciudadanía en general, los que se impliquen y asuman las responsabilidades en su propio desarrollo socioeducativo. Es desde aquí donde la educación social debe proponer el desarrollo de competencias que permitan a los ciudadanos comportarse y sentirse como tales, como miembros de pleno derecho.

\section{Consideraciones Finales}

Para concluir, podemos decir que a medida que ha ido evolucionando el discurso pedagógico-educativo se ha ido produciendo una modificación en el modelo de educación ambiental imperante en cada momento. Por esto, en la actualidad, consideramos que los modelos, como expone Rodríguez (2005), se deben superar con un discurso no sólo integrador entre la Ecología y la Pedagogía, sino entre la Ecología y la Pedagogía Social, que dé lugar a un nuevo modelo de educación ambiental que ella denomina educación socioambiental.

La Educación Social y la Educación Ambiental se presentan, por ello, como praxis social con la misma finalidad. Ambas deben encontrar en la Pedagogía Social fundamentación epistemológica, construcciones teóricas, objetivos, metodologías y contenidos compartidos. Así, los límites no supondrán líneas divisorias entre una y otra práctica, sino que constituirán puntos de encuentros, interrelaciones, bidireccionalidades, enriquecimientos mutuos, aperturas, transdisciplinariedad. Queremos destacar que este se refiere a la unión entre varias materias de forma transversal y que está por sobre todas estas. vale decir su ámbito de acción es superior al de cada una de las disciplinas. Ello evita cotos academicistas alejados de la realidad social/educativa, y que no dan respuestas eficaces a la globalidad y complejidad social, educativa y ambiental.

Consideramos que la transdisciplinariedad, como expone Moraes (2010) implica una actitud del ser humano al vivir un proceso que implica una lógica diferente, una forma compleja de pensamiento sobre la realidad, una percepción más precisa de los fenómenos. Esto implica una actitud de apertura hacia la vida y todos sus procesos. Una actitud que consiste en la curiosidad, la reciprocidad, la intuición de posibles relaciones entre los fenómenos, eventos, cosas y procesos que normalmente escapan a la observación común. Se trata de un principio que requiere que nuestro pensamiento vaya más allá de los aspectos cognitivos, basados en el desarrollo de destrezas y habilidades, de modo a que el proceso educacional pueda, verdaderamente, resonar en la subjetividad de cada aprendiz. Se constituirá como un principio epistemológico que, por su ocurrencia, requiere una actitud de apertura por parte del sujeto implicado, pero cuyo producto trasciende las disciplinas de la Educación Social y la Educación Ambiental, rompiendo las barreras disciplinares, y se presenta desde otro nivel de realidad, en un nivel del proceso de construcción del conocimiento. 
Consecuentemente, entendemos la Educación Social como aquel conjunto de intervenciones socioeducativas que pretende el desarrollo socionatural, individual y colectivo de los sujetos, mediante el conocimiento de una serie de aspectos que faciliten y promuevan la adaptación del individuo a su entorno, para que, posteriormente, sea capaz de desarrollar acciones emancipadoras en y para la mejora socioambiental de la comunidad.

A su vez, la Educación Ambiental debe ser una disciplina cuya intervención debe tener en cuenta los aspectos educativos y sociales ya que las personas, los grupos y las comunidades son los afectados y beneficiarios de las transformaciones en el medio ambiente.

En esta línea, cabría ir centrándose en un modelo alterativo que unifique a ambas materias. Máxime cuando tenemos ante nosotros, una sociedad que, aparentemente, se dirige en sentido opuesto del que pretende la práctica educativa respetuosa con de la década de los ochenta, cuando exponían que la educación ambiental debe promover a la acción, así como inspirar una nueva ética $\mathrm{y}$, en consecuencia, facilitar un cambio de actitudes entre el hombre y la naturaleza, que debe provocar en la práctica nuevas conductas y acciones.

Con posterioridad, y desde una perspectiva alternativa a las posiciones gubernamentales, se manifestaba, en la década de los noventa, en el Foro Global (en la Conferencia Paralela de Río'92), una definición de Educación Ambiental que podemos considerar como precedente del modelo educativo socioambiental, ya que se centra en un acto para la transformación social, y se contempla a la educación como un proceso de aprendizaje permanente basado en el respeto a todas las formas de vida.

De aquí que estemos en el convencimiento de que la Educación Ambiental integrada en la Educación Social, como expone Rodríguez (2005), tiene la posibilidad de extenderse y dar respuesta a las necesidades de sostenibilidad. Si incorporamos la idea de que el bienestar de los individuos no sólo en calidad de vida restringida al ámbito social, sino que dentro del bienestar está también la posibilidad de disfrutar de un medio ambiente equilibrado y armónico, podemos concluir que, dentro de las necesidades básicas de los sujetos, también está explicito la sustentabilidad del entorno.

En este caso, como exponen Amador et al. (2014), la Educación Social completa a la educación general del individuo, teniendo como objeto el logro su madurez social. Convirtiéndose, así, en un aspecto importante de la educación. Esta educación conlleva la formación cívicodemocrática y para la ciudadanía, que desarrollará valores sociales orientados al comportamiento democrático y al compromiso de construcción social.

Igualmente, puntualizar en la idea de que en el concepto de Educación Social está implícita la doble vertiente de la educación en general: desarrollo individual y desarrollo social de los sujetos. Ya que en la medida que el sujeto se desarrolla, la sociedad también lo hace; según el sujeto se transforma, la sociedad se transforma; en definitiva, en tanto en cuanto el sujeto aprende, la sociedad aprende. Es en esta línea y a propósito de los aspectos sociales que llevan al desarrollo, donde consideramos que se hace necesario incluir el término de desarrollo sostenible: ambiental, económico, político, cultural y por supuesto, cívico de la vida del sujeto en la comunidad.

En definitiva, el fin último a seguir en la intervención social que unifique los aspectos tanto educativos como medioambientales, será el de educar a los sujetos hacia el compromiso y la transformación de la sociedad, hacia un desarrollo sostenible tanto ecológico como social. Esta intervención va a requerir de un aprendizaje, mediante el que se consigan una serie de actitudes investigadoras y metodológicas interdisciplinares, para la consecución del conocimiento de la globalidad social, y definir el papel de la educación socioambiental en ella.

Por ello, nuestra finalidad es que podamos llegar a un modelo centrado en lo que nosotros hemos querido denominar intervención educativa socioambiental, que aúne ambas intervenciones, 
para configurar una estructura integrada. Desde esta perspectiva, toda intervención ambiental debe tener en cuenta los aspectos educativos y sociales porque las personas, los grupos y las comunidades son los afectados y beneficiarios de las transformaciones en el entorno físico y toda intervención socioeducativa debe contemplar los aspectos ambientales implicados, puesto que ésta siempre se desarrolla en un contexto socio-físico determinado.

\section{Referencias}

ALVINO, S.; SESSANO, P. (2008). La Educación Ambiental como herramienta para el Ordenamiento Territorial: una experiencia de Política Pública. En: COLOQUIO INTERNACIONAL DE GEOCRÍTICA, 10, 2008, Barcelona. Actas... Barcelona: Universidad de Barcelona, 2008. s/p. Disponible en: < http://www.ub.edu/geocrit/-xcol/116.htm>. Acceso en: 12 feb. 2020.

ARAMBURU, M. Una educación social para un futuro sostenible. Nuevas orientaciones en didáctica de las Ciencias Sociales. Aula: Revista de Pedagogía de la Universidad de Salamanca, Salamanca, s/v, n.13, 63-78, 2001. Disponible en: 〈https://revistas.usal.es/index.php/0214-3402/article/view/3595>. Acceso en: 12 feb. 2020.

ASOCIACIÓN ESTATAL DE EDUCACIÓN SOCIAL - ASEDES. CONGRESO ESTATAL DE LAS EDUCADORAS Y EDUCADORES SOCIALES, 5, 2007, Toledo. Documentos profesionalizadores. Toledo: ASEDES/CGCEES, 2007. p. 1-50. Disponible en: <http://www.eduso.net/archivo/docdow.php?id=143>. Acceso en: 12 feb. 2020.

CARIDE, J. A.; MEIRA, P. A. Educación ambiental y desarrollo humano. Barcelona: Ariel, 2001.

CALVO, S.; CORRALIZA, J.A. Educación Ambiental: conceptos y propuestas. Madrid: CCS, 1994.

ESPAÑA. Constitución Española. Boletín Oficial del Estado, 29 de diciembre de 1978, núm. 311, pp. 29313 a 29424. Disponible en: <https://www.boe.es/eli/es/c/1978/12/27/(1)>. Acceso en: 12 feb. 2020.

ESPAÑA. Ley orgánica 11, de 25 de agosto de 1983. Establece la reforma universitaria. Boletín Oficial del Estado, núm. 209, de 1 de septiembre de 1983, p. 24034 a 24042. Disponible en:

<https://www.boe.es/eli/es/lo/1983/08/25/11>. Acceso en: 12 feb. 2020.

ESPAÑA. Real Decreto 1497/1987, de 27 de noviembre de 1987. Expedición de títulos universitarios oficiales. Boletín Oficial del Estado, núm. 298, de 14 de diciembre de 1987, p. 36639-36643. Disponible en: < https://www.boe.es/boe/dias/1987/12/14/pdfs/A3663936643.pdf>. Acceso en: 12 feb. 2020.

ESPAÑA. Real Decreto 1420/1991, de 30 de agosto de 1991. Establece el título universitario oficial de diplomado en Educación Social. Boletín Oficial del Estado, núm. 243, de 10 de octubre de 1991, p. 32891. Disponible en: <https://www.boe.es/boe/dias/1991/10/10/pdfs/A3289032891.pdf>. Acceso en: 12 feb. 2020.

CUELLO GIJÓN, A. Problemas ambientales y educación ambiental en la escuela. Documento de trabajo para la Estrategia Andaluza de Educación Ambiental. Andaluzía: Centro Nacional de Educación Ambiental. Disponible en: <https://www.miteco.gob.es/es/ceneam/articulos-deopinion/2003_03cuello_tcm30-163448.pdf >. Acceso en: 12 feb. 2020. 
FARACO, J.C. Desde la escuela a la calle: una educación ambiental para el desarrollo comunitario y la participación social. En Cuestiones Pedagógicas: Revista de Ciencias de la Educación, Huelva, s/v, n. 15, p. 78-89, 2001. , Disponible en: <http://institucional.us.es/revistas/cuestiones/15/art_5.pdf>. Acceso en: 12 feb. 2020.

GUTIÉRREZ-PÈREZ, J. La educación ambiental: Fundamentos teóricos, propuestas de transversalidad y orientaciones extracurriculares. Madrid: Editorial La Muralla, 1995.

MINISTERIO DEL MEDIO AMBIENTE. Secretaría General del Medio Ambiente. El Libro Blanco de la Educación Ambiental en Pocas Palabras. Madrid: MMA, 1999. Disponible en: <https://www.miteco.gob.es/es/ceneam/recursos/documentos/pocas_tcm30-77432.pdf>. Acceso en: 12 feb. 2020.

MORAES, M. Transdisciplinariedad y educación. Rizoma Freireano - España, Sevilla, s/v, n. 6, p. 1-19, 2010. Disponible en: <http://www.rizoma-freireano.org/transdisciplinariedad-y-educacion--mariacandida-moraes>. Acceso en: 2 feb. 2020.

MUÑOZ, L. V. A.; ESTEBAN, M. I.; CÁRDENAS-RODRÍGUEZ, R.; TERRÓN-CARO, T. Ámbitos de profesionalización del educador/a social: perspectivas y complejidad. Revista de Humanidades, Sevilla, s/v, n. 21, p. 51-70, Disponible en: <http://www.revistadehumanidades.com/articulos/47-ambitosdeprofesionalizacion-del-educador-a-social-perspectivas-y-complejidad>. Acceso en: 12 feb. 2020.

MUÑOZ, L. V. A.; IBAÑEZ, M. E. Desde la educación social a la educación ambiental. Hacía una intervención educativa socioambiental. Revista de Humanidades, Sevilla, s/v, n. 18, p. 147-160, 2011.

ORGANIZACIÓN MUNDIAL PARA LA SALUD - OMS. Rumbo a un Medio más sano y seguro. Disponible en: <https://www.who.int/es/news-room/commentaries/detail/towards-a-healthier-and-saferenvironment>. Acceso en: 12 feb. 2020.

ORGANIZACIÓN DE LAS NACIONES UNIDAS. División de Desarrollo Sostenible. Fomento de la educación, la capacitación y la toma de conciencia. In: ONU (Org.). Programa 21. Madrid: ONU, 1992. s/p. Disponible en: <https://www.un.org/spanish/esa/sustdev/agenda21/agenda21spchapter36.htm>. Acceso en: 12 feb. 2020.

PASTOR, I. O. Paisaje y educación ambiental. Observatorio Medioambiental, Madrid, s/v, n.3, p. 35-50, 2000. Disponible en: 〈https://core.ac.uk/download/pdf/38809216.pdf>. Acceso en: 2 feb. 2020.

RODRIGO-CAÑO, D.; GUTIÉRREZ, J.; FERRERAS, J. 35 años de éxito de la Educación Ambiental en España. RES Revista de Educación Social, Valencia, s/v, n. 28, p. 32-43, ene./jun. 2019. Disponible en <http://www.eduso.net/res/winarcdoc.php?id=1191>. Acceso en: 12 feb. 2020.

RODRÍGUEZ, M. Introducción a la educación socioambiental desde la Pedagogía Social. Málaga: Aljibe, 2005.

SÁEZ, J. Pedagogía Social y Educación Social. Historia, Profesión y Competencias. Madrid: Pearson, 2007. 
SAUVÉ, L. Perspectivas curriculares para la formación de formadores en Educación Ambiental. En: Reflexiones sobre educación ambiental: artículos publicados en la Carpeta Informativa del CENEAM 2000-2006. Madrid: Ministerio de Medio Ambiente, 2006. p. 219-232.

VIANA-ORTA, M. I. FRANCÉS, I. L.; ZAYAS, B. La formación en trabajo social y educación social en España. Revista de Educación Social, Valencia, s/v, n. 26, p. 34-48, ene./jun., 2018. Disponible en: <http://www.eduso.net/res/winarcdoc.php?id=1101>. Acceso en: 12 feb. 2020. 